\title{
Mathematical Analysis of ESD Generated EM Radiated Fields on Electronic Subsystem
}

\author{
Rajashree Narendra $^{\# 1}$, M.L.Sudheer ${ }^{\# 2}$, V. Jithesh ${ }^{* 3}$, D.C. Pande ${ }^{* 4}$ \\ \# Dept. of ECE, UVCE, Bangalore, India \\ ${ }^{1}$ rajashree.narendra@gmail.com \\ ${ }^{*}$ EMI/EMC Group, LRDE, Bangalore, India
}

\begin{abstract}
Electrostatic Discharge (ESD) generated Electromagnetic (EM) radiated fields can cause malfunctioning or upsets in an electronic system. This paper presents a mathematical computation of the effects of spatial electric fields from an ESD source. These fields may affect the normal functioning of an electronic system. This work uses a MATLAB program to model the response of Very High Frequency (VHF) amplifier to ESD generated radiated EM fields. The ESD source is a Capacitive Spark Discharge (CSD) source. The transient current element has been modeled as a short dipole. The electric field intensity and the magnetic field intensity have been computed by varying distance along the $\theta=\pi / 2$ direction for the CSD current. The frequency spectra of these $E$ and $H$ fields are obtained by taking Discrete Fourier Transform (DFT). The open circuit voltage induced by ESD generated transient fields at the antenna terminals and the voltage coupled to the input of the VHF amplifier fed by antenna is computed by varying distances. The time domain representation of the real part of the voltage at the output terminals of the VHF amplifier is computed by varying distance from the ESD source. Mathematical computation is carried out to predict the effect of the radiated fields on the induced voltages of a subsystem for various distances from the ESD source.
\end{abstract}

Keywords- Electrostatic Discharge; ESD Generated Transient; Electromagnetic radiated fields; Capacitive spark discharge source; VHF Amplifier; Electromagnetic Interference.

\section{INTRODUCTION}

Electrostatic Discharge phenomenon, commonly known as ESD occurs whenever a charged object is grounded: this results in the release and equalization of the static charge. The phenomena that give rise to ESD events may range from collection of charged particles due to ambient plasma, to the generation of charges due to triboelectric effect. This release of electric charge results in radiated electromagnetic fields that may couple to neighboring electrical circuits causing electromagnetic interference.

ESD can be a serious threat to electronic equipment. There is a need for ESD prevention and design of ESD immune electronics. Lot of effort has gone into the study of ESD current waveforms in order to develop discharge simulators for simplifying and standardizing ESD susceptibility testing [1, 2, 3]. The radiated fields associated with ESD events [4] have received much less attention compared to conducted susceptibility $[5,6]$.

This paper predicts the ESD response of the front end of the radio frequency subsystems working in Very High frequency (VHF) band. The ESD simulation current is generated using a capacitive spark discharge source. The ESD event takes place at specific distances from the antenna connected to the electronic subsystem. This work is modeled on similar computation presented by Shastry and Hariharan [7] for fields at 1-meter distance from the ESD source. The work presented in this paper looks into the response of the VHF amplifier to the radiated EM fields due to an ESD event for distances from $0.5 \mathrm{~m}$ to $4.0 \mathrm{~m}$.

\section{TRANSIENT CURRENT SOURCE}

The ESD current waveform used in this study is based on the CSD model described by Norberg et.al. [8]. This model assumes that the discharge current $i(t)$ may be expressed in the form:

$$
i(t)=P e^{-q t} \sin (s t)
$$

where the values of $P, q, s$ are selected as mentioned in reference [7] for a simple current waveform for the CSD from an ESD simulator and the current pulse shown in Fig. 1 fits the pulse shape obtained by (1) when $P=90$ Amps, $q=1.5 \times 10^{7}$ and $s=\pi \times 10^{7}$. The ESD waveform is not modeled on any real event. More realistic models based on IEC 61000-4-2 standard like the equivalent circuit models and full wave models $[9,10$, $11,12]$ will be considered for future work.

The transient current flowing through the object under test may be represented by a Z-oriented current element located with its center at the origin of the Cartesian coordinate system. Since this is a preliminary work, a Hertzian Dipole model was adopted though it is a highly simplified model for simulating fields of ESD. We have not considered the fact that the radiator is not just the arc, but the entire circuit formed by the simulator, discharge head, target and the return strap [13]. The advanced full wave models for system level coupling simulation [13] will be considered in future.

\section{RADIATED TRANSIENT FIELDS}

The field component at any point $P(r, \theta, \phi)$ of the short dipole [7] is expressed in terms of spherical coordinates $r, \theta$ and $\phi$ are as follows:

$$
E_{r}(t)=\frac{L \operatorname{Cos} \theta}{2 \pi}\left(\eta_{0} \frac{i(t)}{r^{2}}+\frac{\int i(t) d t}{\varepsilon_{0} r^{3}}\right)
$$




$$
\begin{gathered}
E_{\theta}(t)=\frac{L \operatorname{Sin} \theta}{4 \pi}\left(\frac{\mu_{0}}{r} \frac{d}{d t} i(t)+\eta_{0} \frac{i(t)}{r^{2}}+\frac{\int i(t) d t}{\varepsilon_{0} r^{3}}\right) \\
H_{\phi}(t)=\frac{L \operatorname{Sin} \theta}{4 \pi}\left(\frac{1}{r c} \frac{d}{d t} i(t)+\frac{i(t)}{r^{2}}\right)
\end{gathered}
$$

where

$\mu_{\mathrm{o}}=4 \pi \times 10^{-7} \mathrm{H} / \mathrm{m}=$ permeability of free space

$\varepsilon_{\mathrm{o}}=(1 / 36 \pi) \times 10^{-9} \mathrm{~F} / \mathrm{m}=$ permittivity of free space

$c=3 \times 10^{8} \mathrm{~m} / \mathrm{s}=$ velocity of light in free space

$\eta_{0}=120 \pi=$ intrinsic impedance of free space

$L=$ Length of the current element

$t=t^{\prime}-r / c=$ time variable for retarded current

$t^{\prime}=$ time variable

The field intensities along the direction $\theta=\pi / 2$ have been computed for various distances $(r=1.5,2,2.5,3 m)$ for $0 \leq \mathrm{t}$ $\leq 400 \mathrm{~ns}$. The corresponding frequency spectra of the $E$ and $H$ fields are given in Fig. $2 \&$ Fig. 3. These are obtained by taking a 2000 point DFT of the sample points obtained using (3) \& (4) with $\theta=\pi / 2, L=10 \mathrm{~mm}$ and $r=1.5,2,2.5,3 \mathrm{~m}$. From the spectra we observe that a greater part of the energy due to ESD simulation currents has frequency components extending to the VHF and UHF bands.

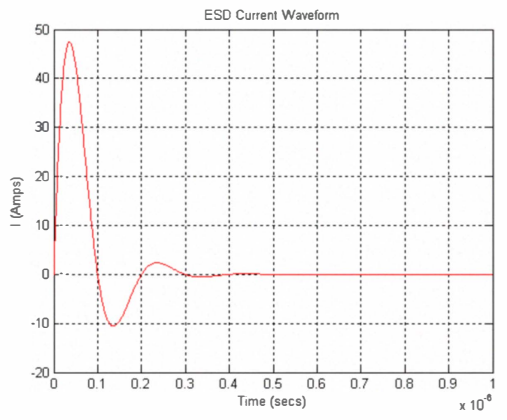

Figure 1. ESD Current Waveform

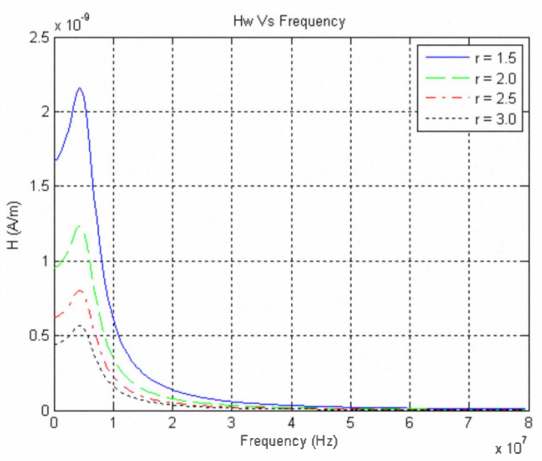

Figure 2. Frequency Spectrum of H-field

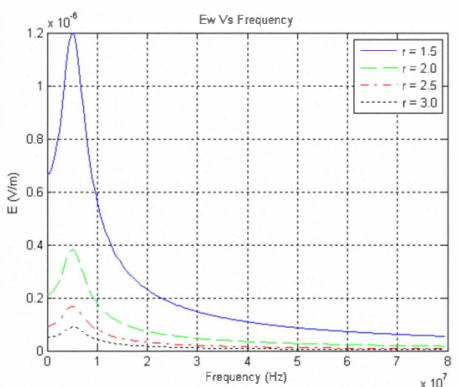

Figure 3. Frequency Spectrum of E-field

\section{RADIATED FIELD TO ANTENNA COUPLING MODEL}

The open circuit voltage $V_{o c}$ induced by ESD generated radiated fields at the antenna terminals are found using the relation

$$
V_{o c}=\bar{h} \cdot \bar{E}^{i}
$$

where $\bar{h}=$ vector effective height and $\bar{E}^{\mathrm{i}}=$ electric field at antenna terminals.

Assuming the vector effective height of the antenna is parallel to the polarization of the incident $E$-field and the antenna terminals are located at $\theta=\pi / 2$, the frequency spectrum of the open circuit voltage induced at antenna terminals, is expressed as

$$
V_{o c}(\omega)=h_{e}(\omega) E_{\theta}(\omega)
$$

where $h_{e}(\omega)=$ angular frequency dependent complex effective height

$E_{\theta}(\omega)=$ frequency spectrum of $E$-field at $\theta=\pi / 2$

The published data of $h_{e}(\omega)$ [14] for a UHF/VHF communication antenna AT-1076 (operating frequency band $225-400 \mathrm{MHz}$ ) is used along with a spline interpolation for computing the effective height. The frequency spectra of the open circuit voltage induced at the antenna input terminals for distances $r=1.5,2,2.5,3 m$ by using the $h_{e}(\omega)$ data is shown in Fig. 4.

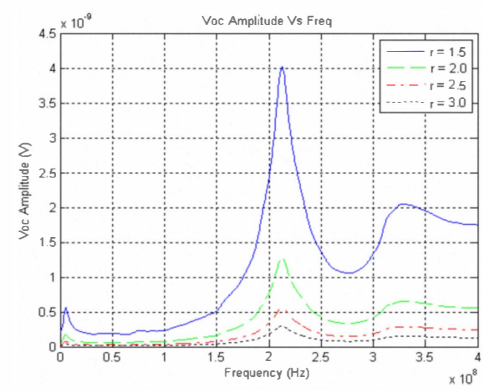

Figure 4. Magnitude of frequency spectrum of open circuit voltage coupled to the antenna terminals 


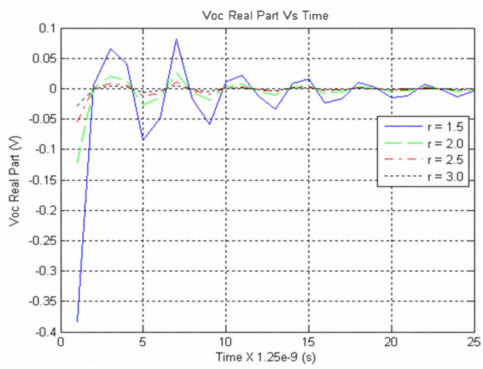

Figure 5. Real part of open circuit voltage coupled to antenna terminals

The time domain representation of the magnitude of the induced open circuit voltage is obtained by taking 2000-point Inverse DFT of Voc $(\omega)$. The magnitude of the induced open circuit voltage is plotted for various distances $(r=1.5,2,2.5$, $3 \mathrm{~m})$ and is shown in Fig. 5.

\section{ANTENNA TO AMPLIFIER COUPLING MODEL}

The voltage coupled to the input of the VHF amplifier fed by the antenna depends on the antenna impedance and input impedance of the amplifier. The input voltage (without considering the cable effect) is given by

$$
V_{\text {in }}(\omega)=\frac{Z_{\text {in }}}{Z_{\text {in }}+Z_{a}} V_{o c}(\omega)
$$

$Z_{\text {in }}=$ input impedance of the VHF amplifier (assumed to be $50 \Omega$ amplifier), $Z_{a}=$ antenna impedance

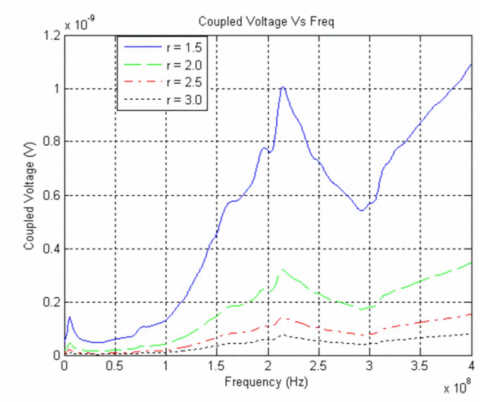

Figure 6. Magnitude of frequency spectrum of voltage coupled to the amplifier input

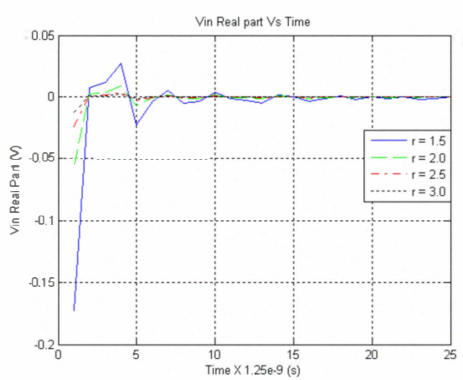

Figure 7. Real part of voltage coupled to the amplifier input
Using the published data on $Z_{a}$ [14], a spline interpolation routine has been written for computing $Z_{a}$ in the $30 \mathrm{MHz}$ to 300 $\mathrm{MH}_{\mathrm{Z}}$ band. The spectrum of $V_{\text {in }}(\omega)$ is computed for $r=1.5,2$, $2.5,3 \mathrm{~m}$ and is shown in Fig. 6 . The time domain representation of the real part of the voltage coupled to the amplifier input terminals for distances $(r=1.5,2,2.5,3 m)$ is plotted in Fig. 7 . If the distance between the ESD generator and the antenna is increased or decreased, this peak magnitude also changes accordingly.

\section{AMPLIFIER MODEL}

The frequency spectrum of the voltage at the amplifier output terminals is given by

$$
V_{\text {out }}(\omega)=A_{v}(\omega) V_{\text {in }}(\omega)
$$

where $A_{v}(\omega)$ is the voltage gain of the amplifier stage.

An n-channel dual gate MOSFET (3N187) was chosen as an active device to construct the RF amplifier and a set of Yparameters (for desired operating point to give most suitable self bias for the device) was taken from data sheets in reference [10]. For a single stage amplifier, the voltage gain expressed in terms of the Y-parameters of the active device and the load admittance is given by

$$
A_{v}(\omega)=\frac{Y_{F}}{Y_{0}+Y_{L}}
$$

where $Y_{F}=$ short circuit forward transfer admittance, $Y_{O}=$ Short circuit output admittance and $Y_{L}=$ load admittance.

The RF amplifier used has a centre frequency of $232 \mathrm{MHz}$ and a bandwidth of $10 \mathrm{MHz}$. The voltage gain curve for the amplifier has been plotted in the Fig. 8 using the data given in [15]. The saturation of the amplifier was not taken into account. The characteristics and nonlinear effects can be considered in future work.

The magnitude of frequency spectrum of the output voltage of the amplifier for $r=1.5,2,2.5,3 m$ shown in Fig. 9 is obtained from (8) and the voltage gain curve for the dual gate MOSFET VHF amplifier. The time domain representation of the real part of the amplifier output voltage for distances $(r=$ $1.5,2,2.5,3 m)$ is shown in Fig. 10.

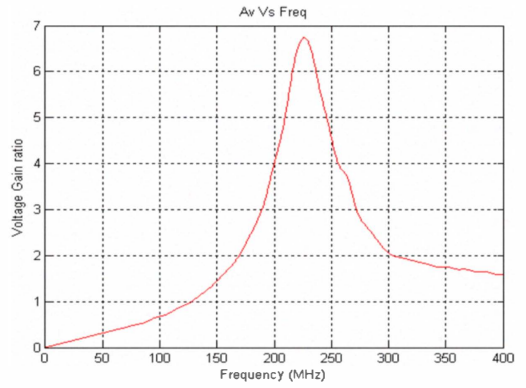

Figure 8. Voltage gain curve for the dual gate MOSFET VHF amplifier 


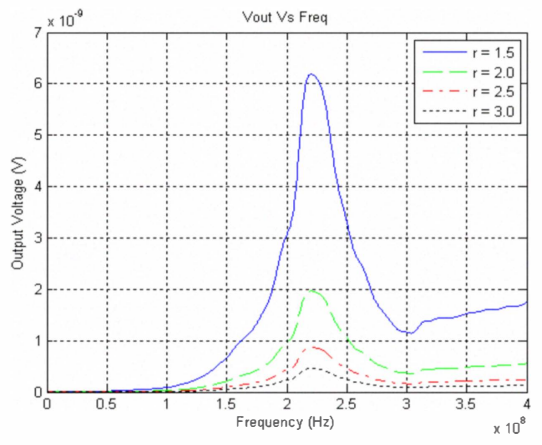

Figure 9. Magnitude of frequency spectrum of output voltage of the amplifier

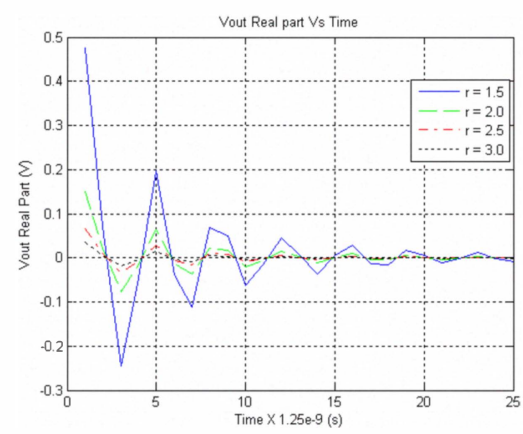

Figure 10. Real part of amplifier output voltage

\section{ANALYSIS}

The analysis was carried out for distances ranging from 0.5 to $4.0 \mathrm{~m}$ and the values are listed in Table 1 . The amplitude of the response decreases sharply with increase in distance from the ESD source. Experiments are being conducted to study the effects of radiated EM field from an ESD event on the functioning of integrated circuits and systems. These results will be presented shortly. The complex circuit analysis using the SPICE simulation is also in progress.

TABle 1: PeaK Values (ABsolute) For Various Distances.

\begin{tabular}{|l|l|l|l|l|l|}
\hline $\begin{array}{c}\text { Distance } \\
(\mathrm{m})\end{array}$ & $\begin{array}{c}\mathrm{E} \\
(\mathrm{V} / \mathrm{m})\end{array}$ & $\mathrm{H}(\mathrm{A} / \mathrm{m})$ & $\begin{array}{c}\text { Voc } \\
(\text { volts })\end{array}$ & $\begin{array}{c}\text { Vin } \\
(\text { volts })\end{array}$ & $\begin{array}{c}\text { Vout } \\
(\text { volts })\end{array}$ \\
\hline 0.5 & 575.74 & 0.1513 & 16.474 & 7.446 & 20.506 \\
\hline 1.0 & 101.97 & 0.0380 & 2.918 & 1.319 & 3.632 \\
\hline 1.5 & 13.37 & 0.0171 & 0.383 & 0.173 & 0.476 \\
\hline 2.0 & 4.25 & 0.0097 & 0.122 & 0.055 & 0.151 \\
\hline 2.5 & 1.88 & 0.0063 & 0.054 & 0.024 & 0.067 \\
\hline 3.0 & 0.99 & 0.0045 & 0.028 & 0.013 & 0.035 \\
\hline 3.5 & 0.59 & 0.0034 & 0.017 & 0.008 & 0.021 \\
\hline 4.0 & 0.37 & 0.0027 & 0.011 & 0.005 & 0.013 \\
\hline
\end{tabular}

\section{CONCLUSION}

The modeling of the response of a VHF amplifier to an ESD generated EM radiated field is done using a standard approach. The results obtained for a distance of 1-metre from the ESD source correlate to the published results. This paper analyses the effect of radiated EM field on a VHF amplifier: the coupling of voltage into the antenna terminals, from the antenna terminals to the amplifier input and finally the amplifier response.

\section{ACKNOWLEDGEMENT}

The first author [Rajashree Narendra] would like to thank BNM Institute of Technology, (where she is currently the Professor and Head of Telecommunication Department) for their constant encouragement in her doctoral research work. Also she would like to thank the EMI/EMC group at LRDE for providing an opportunity to carry out her $\mathrm{PhD}$ research work.

\section{REFERENCES}

[1] P. Richman and A. Tasker, "ESD Testing: The Interface Between Simulator And Equipment Under Test", Proceedings of $6^{\text {th }}$ International Zurich Symposium Technical Exhibition, EMC, March 1985, pp-25-30.

[2] A. Tasker, "ESD Discharge Waveform Measurements: The First Step In Human ESD Simulation", Proceedings of IEEE International Symposium EMC, Wakefield, MA, August 1985, pp 246-25.

[3] James E. Vinson and Juin J. Ziou, "Electrostatic Discharge In Semiconductor Devices: An Overview", Proceedings of the IEEE, Vol. 86, No 2, February 1998, pp 399-418.

[4] P.F. Wilson, M.T. Ma and A.R. Ondrejka, "Fields Radiated By Electrostatic Discharges", IEEE International Symposium on EMC, Aug 1988, pp 179-183.

[5] M.L. Sudheer, M.K. Gunashekaran, "Analysis Of Rectifier Failure To Power Line Transients"' European Power Electronics (EPE) Journal, Vol. 9, No.1-2, October 1999.

[6] M.L. Sudheer, M.K. Gunashekaran, "Effects Of Common Mode Transients On Front End Rectifiers In Off Line SMPS", IEEE International Symposium on EMC, August 2005, Chicago, USA.

[7] S.V.K. Shastry and V.K. Hariharan, "Computer Aided Analysis Of ESD Effects In Dual Gate MOSFET VHF Amplifier", IEEE International Symposium on EMC, Aug 1990, pp. 424-430

[8] Anna Norberg, V. Szedenik and S. Lundquist, "On The Pulse Shape Of Discharge Currents", Journal of Electrostatics, 1989, pp. 79-88.

[9] H.Tanaka, O.Fujiwara and Y.Yamanaka, "A Circuit Approach to simulate Discharge Current Injected in Contact with an ESD-gun," Int. Sym. on EMC, May 2002, pp. 486-489.

[10] S.Caniggia nad F.Maradei, "Circuital and Numerical Modeling of Electrostatic Discharge Generators," Industry Applications Conference, October 2005, pp. 1119-1123.

[11] Cai Qing, Jayong Koo, Argha Nandy, Jong Sung Lee and Byong Su Seol, "Advanced Full Wave ESD Generator Model for System Level Coupling Simulation," EOS/ESD Symposium, 2008.

[12] David Pommerenke and Martin Aidam, "To What Extent do Contactmode and Indirect ESD Test Methods Reproduce Reality," EOS/ESD Symposium, Semptember 1995, pp. 101-109.

[13] Michael Mardiguian, "Comments on Fields Radiated by Electrostatic Discharge," IEEE Trans. On EMC, Vol 34, No. 1, February 1992, pp. 62.

[14] Y.T. Lo and S.W. Lee (Editors), "Antenna Hand Book Theory: Application And Design", Van Nostrand Reinhold Co N.Y., 1988, pp. 30.21.

[15] Amy Purushotham and S.V.K Shastry, "CAD Optimizes The Gain Of Dual Gate MOSFET VHF Amplifier," RF Design, February 1989, pp. $61-72$ 